\title{
SALUD MENTAL EN ADULTOS MAYORES ATENDIDOS EN LA RED HOSPITALARIA PÚBLICA DE MEDELLÎN, COLOMBIA
}

\author{
Andrés A. Agudelo-Suárez ${ }^{1, a}$, Adriana Posada-López ${ }^{1,2, b}$, Edwin J. Meneses-Gómez²,c
}

\begin{abstract}
RESUMEN
Objetivos. Determinar la prevalencia de mala salud mental y sus factores asociados en adultos mayores atendidos en la Empresa Social del Estado (ESE) Metrosalud. Materiales y métodos. Estudio transversal mediante muestreo bietápico en las unidades hospitalarias y centros de salud adscritos a la red de la ESE Metrosalud de Medellín. Participaron 342 adultos de 65 y más años (58,2\% mujeres). Variables: mala salud mental (medida con el GHQ12), apoyo social (Duke-11); sociodemográficas: edad, sexo, estrato socioeconómico, nivel educativo, zona de residencia, estado civil, y ocupación. Se describieron las variables del estudio, se calculó la prevalencia total de acuerdo con las variables sociodemográficas, y se estimó la asociación de mala salud mental de acuerdo con variables seleccionadas calculando Odds Ratio con sus intervalos de confianza al 95\% ajustado por posibles variables confusoras mediante regresión logística. Resultados. La población adulta mayor sin estudios de ambos sexos (ORa 7,61; IC 95\%: 2,49-23,34), las mujeres viudas (ORa 2,78; IC 95\%: 1,21-6,40) y los hombres y mujeres con bajo apoyo social (ORa 3,05; IC 95\%: 1,65$5,66)$, reportaron mayor riesgo de mala salud mental, con respecto a sus contrapartes de referencia. Conclusiones. Se encontró una alta prevalencia de mala salud mental en la población estudiada, con diferencias de acuerdo con factores sociodemográficos, lo que sugiere la situación de vulnerabilidad social en este colectivo que impacta en su situación de salud. Se requieren estrategias y políticas sociales y de salud que contribuyan al bienestar de este grupo poblacional.
\end{abstract}

Palabras clave: Salud mental; Adulto mayor; Perfil de salud; Encuestas de salud (fuente: DeCS BIREME).

\section{MENTAL HEALTH IN OLDER ADULTS OF A PUBLIC HOSPITAL NETWORK OF MEDELLIN, COLOMBIA}

\begin{abstract}
Objectives. To determine the prevalence of poor mental health and associated factors in older adults of the Social Enterprise of the State (ESE) Metrosalud. Materials and methods. Cross-sectional study using two-stage sampling in hospital units and health centers in the network of the ESE Metrosalud of Medellin. Participants included 342 adults aged 65 and over (57.8\% women). Variables: poor mental health (measured with the GHQ12), social support (Duke-11); sociodemographic: age, sex, socioeconomic status, educational level, place of residence, marital status and occupation. Study variables were described, the overall prevalence was calculated according to sociodemographic variables. The association of poor mental health with selected variables was estimated by calculating odds ratios with $95 \%$ confidence intervals adjusted for possible confounding variables using logistic regression. Results. The senior population without education in both sexes (ORa = 7.61; $95 \% \mathrm{Cl}$ : 2.49 to 23.34 ), widows (ORa $2.78 ; 95 \% \mathrm{Cl}: 1.21$ to 6.40 ) and men and women with low social support (ORa $3.05 ; 95 \% \mathrm{Cl}: 1.65-5.66)$, reported increased risk of poor mental health, compared to their counterparts of reference. Conclusions. We found a high prevalence of poor mental health in the population studied, with differences according to socio-demographic factors, suggesting that the social vulnerability in this group impacts their health status. Health and social policies and strategies that contribute to the welfare of this population group are required.
\end{abstract}

Key words: Mental health; Elderly; Health profile; Health surveys (source: MeSH NLM).

\section{INTRODUCCIÓN}

La población adulta mayor se constituye desde hace varias décadas en un grupo poblacional representativo y sensible en términos demográficos y sociales ${ }^{(1-3)}$. En primer lugar, porque se han dado profundos cambios en la dinámica poblacional mundial, en Colombia se estima que para el año 2020 el grupo de 65 o más años ocupará un $7,6 \%$ de la población total de hombres y un $9,4 \%$ de la población de mujeres ${ }^{(4)}$. Esto también se proyecta para Medellín, la segunda ciudad de Colombia, con una población cercana a los dos millones y medio de habitantes ${ }^{(5)}$, donde ha llamado la atención el fenómeno de envejecimiento de la población ${ }^{(6)}$. En

\footnotetext{
Facultad de Odontología, Universidad de Antioquia. Medellín, Colombia.

Facultad de Odontología, Fundación Universitaria Autónoma de las Américas. Medellín, Colombia.

a Odontólogo, especialista en administración en servicios de salud, Phd en Salud Pública; ${ }^{b}$ odontóloga, licenciada en biología y química; ${ }^{c}$ odontólogo, especialista en programas preventivos bucodentales a nivel individual y público. Phd en ciencias odontológicas. Recibido: 20-01-15 Aprobado: 05-08-15
} 
segundo lugar, las investigaciones realizadas hasta el momento evidencian notoriamente la condición de la población adulta mayor, ya que se encuentra expuesta a situaciones de vulnerabilidad social, dificultades de acceso a servicios sociales y de salud y baja percepción de su calidad de vida ${ }^{(7-9)}$.

En cuanto a las condiciones de salud para esta población, la literatura científica se hace notoria sobre aspectos relacionados con el proceso fisiológico propio del envejecimiento, que ocasionan un estado de fragilidad (10), deterioro cognitivo ${ }^{(11)}$, situación de dependencia funcional ${ }^{(12)}$, entre otros. Por otro lado, existen determinantes sociales que impactan en las desigualdades y en el bienestar de esas personas; por ejemplo, un estudio realizado en Cataluña (España), destaca la red social y el apoyo social como elementos claves relacionados con la salud mental en los adultos mayores no institucionalizados ${ }^{(13)}$.

Específicamente, en el componente de la salud mental, la prevalencia de mala salud en la población adulta mayor es variable y depende entre otras características, del tipo de estudio, del indicador utilizado y del instrumento de recolección de información. Según la OMS ${ }^{(14)}$, un 15\% de los adultos de 60 años o mayores sufren algún trastorno mental. Una revisión sistemática realizada en la Unión Europea ${ }^{(15)}$, encontró que los síntomas más prevalentes relacionados con alteraciones de salud mental son la depresión y la demencia, llamando la atención sobre la necesidad de impulsar mayores estudios epidemiológicos coordinados, para facilitar los sistemas de vigilancia epidemiológica y tener datos comparables.

En Colombia, la última Encuesta Nacional de Demografía y Salud ${ }^{(16)}$, utiliza elementos del test de depresión y ansiedad de Zung, y evidencia que una buena parte de la población ha manifestado síntomas sugerentes de mala salud mental. Para la ciudad de Medellín, si bien existen estudios que abordan componentes importantes de la calidad de vida, el bienestar social y el apoyo social como elementos determinantes de la situación de salud para la población adulta mayor ${ }^{(6-9,17)}$, los estudios específicos en salud mental aún son escasos, especialmente en población no institucionalizada. Adicionalmente, se requiere profundizar en algunos aspectos epidemiológicos desde el abordaje de los determinantes sociales que podrían explicar diversas situaciones de desigualdad y vulnerabilidad social. Se tiene como referencia un estudio realizado en Centros de Bienestar del Anciano (CBA) en la ciudad (18), pero es importante abordar otros indicadores. Conocer la situación en salud mental es un paso muy importante para la realización de políticas y estrategias basadas en la realidad social que vive la población adulta de la segunda ciudad más importante de Colombia.
Teniendo en cuenta lo anterior, el objetivo de este estudio fue determinar la prevalencia de mala salud mental en adultos mayores atendidos en red pública hospitalaria del municipio de Medellín, Empresa Social del Estado Metrosalud (ESE Metrosalud), así como sus factores relacionados.

\section{MATERIALES Y MÉTODOS}

Se realizó un estudio transversal. La población de estudio la constituyeron los adultos mayores de la ESE Metrosalud que acuden a los servicios de consulta externa en su red de servicios de Medellín. Metrosalud es la red pública hospitalaria de primer y segundo nivel que brinda servicios de salud a un segmento poblacional importante de la ciudad, especialmente personas de estratos socioeconómicos bajos y afiliados al régimen subsidiado en salud (personas sin capacidad de pago) ${ }^{(19)}$. Se tuvieron en cuenta para este estudio a hombres y mujeres con 65 o más años y que fueron seleccionados a partir de los registros de consulta de primera vez a cargo de la unidad de estadística de la Institución para el año 2011. Se excluyeron aquellas personas en situación de discapacidad auditiva o visual o con compromiso sistémico que les impidiera asistir a la institución, ya que no se tuvo en cuenta la atención domiciliaria y a las personas que no aceptaron participar en el estudio.

Según las características de la institución, se realizó un muestreo en dos etapas. En un primer momento se seleccionaron las unidades prestadoras y los centros de salud que participarían en la muestra y, posteriormente, se asignó la muestra proporcionalmente en cada uno de estas unidades y centros. Se determinó inicialmente una muestra total de 354 adultos mayores, definida de acuerdo con otros estudios en calidad de vida y salud bucal, dentro del proyecto macro realizado en la institución ${ }^{(20,21)}$. Para este análisis se usó la información de 342 adultos mayores (58,2\% mujeres), lo cual constituye un $97 \%$ de la muestra total, luego de hacer depuración de los datos.

Brevemente, el proyecto más amplio contempló la utilización de una encuesta estructurada y un examen odontológico completo, con información sobre datos sociodemográficos, variables de salud autopercibida (mental, general y bucal), utilización de servicios de salud bucal, calidad de vida relacionada con la salud bucal, examen de articulación temporo-mandibular, mucosa bucal, evaluación de tejidos blandos, examen periodontal, dental y protésico. Estas fueron realizadas por un grupo de trabajo de campo (dos examinadores y cuatro encuestadores), los cuales fueron capacitados y calibrados para obtener encuestas de calidad. Se realizó una prueba piloto en diez adultos mayores, con el fin de 
corregir, revisar el lenguaje, la adaptación cultural de las preguntas y la consistencia general de los instrumentos de recogida de información. El trabajo de campo se realizó entre los meses de marzo y diciembre de 2013.

Concretamente, en este artículo se utilizaron las siguientes variables: edad (65-74, $\geq 75$ ); nivel educativo (sin estudios, primaria, secundaria-universitaria); estrato socioeconómico (este fue definido según la clasificación dada en la vivienda por la empresa de servicios públicos domiciliarios de la ciudad y categorizada en bajo $-0,1$ y 2- y medio -3 y 4-); estado civil (soltero, casado/unión libre, viudo, separado); ocupación (esta fue obtenida de la clasificación internacional de ocupaciones adaptada para Colombia ${ }^{(22)}$ y se categorizó en no manual (directivas, profesionales universitarios, científicos e intelectuales, técnicos, postsecundarios no universitarios y asistentes, empleados de oficina, trabajadores de los servicios y vendedores (comerciantes), manual (agricultura, manuales de la industria y de la construcción y trabajadores no calificados), labores del hogar y otras (jubilados y estudiantes); zona de residencia (urbana, rural).

Para evaluar la dimensión apoyo social se utilizó el perfil de Duke-11, el cual es un cuestionario o escala compuesta de ocho frases que se puntúan según cinco categorías de escala de Likert, que van desde «mucho menos de lo que deseo» (que puntúa 1) a «tanto como deseo» (que puntúa 5). Para obtener las puntuaciones de la escala se suman los valores obtenidos a cada una de las frases y el rango de puntuación oscila entre 11 y 55 puntos, a mayor puntaje mayor apoyo percibido. Se optó por un punto de corte en una puntuación < 32, para considerar el apoyo social percibido como bajo ${ }^{(23,24)}$.

El componente de salud mental se midió con el General Health Questionnaire de 12 preguntas (GHQ-12) (25), que es un cuestionario que tiene la finalidad de realizar un tamiz de los trastornos mentales actuales y no tiene como objetivos realizar diagnósticos clínicos ni valorar trastornos crónicos. Se pregunta en forma de escala de Likert de cuatro categorías si la persona ha experimentado recientemente un síntoma en particular. $A$ las cuatro categorías de respuesta se les adjudicó el valor 0 (respuestas 1 y 2) o 1 (respuestas 3 y 4). Se consideró como mala salud mental a la persona con un punto de corte $\geq 3$, de acuerdo con otros estudios en poblaciones de contextos similares al de la ciudad de Medellín y con el fin de lograr comparabilidad internacional ${ }^{(13)}$.

Los análisis se realizaron separadamente para hombres y mujeres y en forma conjunta. En primer lugar, se describieron las variables del estudio mediante frecuencias absolutas y relativas. Posteriormente, se calculó la prevalencia de mala salud mental con sus intervalos de confianza al $95 \%$ para cada una de las variables seleccionadas. Por último, se estudió la asociación entre mala salud mental y las variables nivel educativo, estado civil, zona de residencia y apoyo social percibido calculando Odds Ratio (OR) crudas y ajustadas por medio de regresión logística, con sus intervalos de confianza al 95\%. Para este estudio se muestran los modelos ajustados por las variables sociodemográficas.

Este estudio cumple con los requisitos éticos para investigación en seres humanos de acuerdo con la normativa internacional y nacional. Se solicitó el consentimiento para participar en el estudio a los adultos mayores, garantizando la confidencialidad y el respeto por la información. Este estudio fue aprobado por el Comité de Ética de Investigación (CEI) de la institución METROSALUD, según acta 09-2011.

\section{RESULTADOS}

En la Tabla 1 se presentan las características sociodemográficas de la población participante en el estudio. En términos generales el $69 \%$ eran menores de 75 años, el $92 \%$ con estudios primarios o sin estudios,

Tabla 1. Características sociodemográficas de la población adulta mayor participante en el estudio. E.S.E METROSALUD. Medellín, Colombia. 2013 (n=342)

\begin{tabular}{|c|c|c|c|c|c|}
\hline \multirow{2}{*}{ Características } & \multicolumn{2}{|c|}{ Hombres } & \multicolumn{2}{|c|}{ Mujeres } & Total \\
\hline & $\mathbf{n}$ & $(\%)$ & $\mathbf{n}$ & $(\%)$ & n $\quad(\%)$ \\
\hline \multicolumn{6}{|l|}{ Edad (años) } \\
\hline $65-74$ & 91 & $(63,6)$ & 144 & $(72,4)$ & $235(68,7)$ \\
\hline$\geq 75$ & 52 & $(36,4)$ & 55 & $(27,6)$ & $107(31,3)$ \\
\hline \multicolumn{6}{|l|}{ Nivel educativo } \\
\hline Sin estudios & 48 & $(33,6)$ & 65 & $(32,7)$ & $113(33,0)$ \\
\hline Primaria & 82 & $(57,3)$ & 121 & $(60,8)$ & $203(59,4)$ \\
\hline Secundaria-Universitaria & 13 & $(9,1)$ & 13 & $(6,5)$ & $26(7,6)$ \\
\hline \multicolumn{6}{|c|}{ Estrato socioeconómico } \\
\hline Bajo (1-2) & 124 & $(86,7)$ & 169 & $(84,9)$ & $293(85,7)$ \\
\hline Medio (3-4) & 19 & $(13,3)$ & 30 & $(15,1)$ & $49(14,3)$ \\
\hline \multicolumn{6}{|l|}{ Ocupación } \\
\hline No manual & 3 & $(2,1)$ & 1 & $(0,5)$ & $4 \quad(1,2)$ \\
\hline Manual & 47 & $(32,9)$ & 16 & $(8,0)$ & $63(18,4)$ \\
\hline Labores del hogar & 8 & $(5,6)$ & 172 & $(86,4)$ & $180(52,6)$ \\
\hline Otros & 85 & $(59,4)$ & 10 & $(5,0)$ & $95(27,8)$ \\
\hline \multicolumn{6}{|l|}{ Estado civil } \\
\hline Soltero & 23 & $(16,1)$ & 52 & $(26,1)$ & $75(21,9)$ \\
\hline Casado-Unión libre & 84 & $(58,7)$ & 64 & $(32,2)$ & $148(43,3)$ \\
\hline Viudo & 24 & $(16,8)$ & 66 & $(33,2)$ & $90(26,3)$ \\
\hline Separado & 12 & $(8,4)$ & 17 & $(8,5)$ & $29(8,5)$ \\
\hline \multicolumn{6}{|l|}{ Zona de residencia } \\
\hline Urbana & 129 & $(90,2)$ & 177 & $(88,9)$ & $306(89,5)$ \\
\hline Rural & 14 & $(9,8)$ & 22 & $(11,1)$ & $36(10,5)$ \\
\hline \multicolumn{6}{|l|}{ Apoyo social } \\
\hline Normal & 109 & $(76,2)$ & 152 & $(76,4)$ & $261(76,3)$ \\
\hline Bajo & 34 & $(23,8)$ & 47 & $(23,6)$ & $81(23,7)$ \\
\hline Total & 143 & $(41,8)$ & 199 & $(58,2)$ & $342(100)$ \\
\hline
\end{tabular}


Tabla 2. Prevalencia de mala salud mental en la población adulta mayor atendida en la E.S.E METROSALUD de acuerdo a características sociodemográficas. Medellín, Colombia. $2013(n=342)$

\begin{tabular}{|c|c|c|c|c|c|c|c|c|c|}
\hline \multirow{2}{*}{ Características } & \multicolumn{3}{|c|}{ Hombres } & \multicolumn{3}{|c|}{ Mujeres } & \multicolumn{3}{|c|}{ Total } \\
\hline & $\mathbf{n}$ & $\%($ IC $95 \%)$ & $\begin{array}{c}\text { Valor } \\
p^{*}\end{array}$ & $\mathbf{n}$ & $\%$ (IC 95\%) & $\begin{array}{c}\text { Valor } \\
p^{*}\end{array}$ & $\mathbf{n}$ & $\%$ (IC 95\%) & $\begin{array}{c}\text { Valor } \\
p^{*}\end{array}$ \\
\hline \multicolumn{10}{|l|}{ Edad en años } \\
\hline $65-74$ & 54 & $59,3(48,7-70,0)$ & 0,627 & 89 & $61,8(53,5-70,1)$ & 0,149 & 143 & $60,9(54,4-67,3)$ & 0,190 \\
\hline$\geq 75$ & 33 & $63,5(49,4-77,5)$ & & 40 & $72,7(60,0-85,4)$ & & 73 & $68,2(58,9-77,5)$ & \\
\hline \multicolumn{10}{|l|}{ Nivel educativo } \\
\hline Sin estudios & 32 & $66,7(52,3-81,0)$ & 0,013 & 49 & $75,4(64,1-86,6)$ & 0,007 & 81 & $71,7(62,9-80,4)$ & $<0,001$ \\
\hline Primaria & 52 & $63,4(52,4-74,5)$ & & 76 & $62,8(53,8-71,8)$ & & 128 & $63,1(56,2-69,9)$ & \\
\hline Secundaria- Universitaria & 3 & $23,1(5,0-53,8)$ & & 4 & $30,8(9,1-61,4)$ & & 7 & $26,9(8,0-45,9)$ & \\
\hline \multicolumn{10}{|l|}{ Estrato socioeconómico } \\
\hline Bajo (1-2) & 78 & $62,9(54,0-71,8)$ & 0,196 & 110 & $65,1(57,6-72,6)$ & 0,853 & 188 & $64,2(58,5-69,8)$ & 0,346 \\
\hline Medio (3-4) & 9 & $47,4(24,4-71,1)$ & & 19 & $63,3(44,4-82,2)$ & & 28 & $57,1(42,3-72,0)$ & \\
\hline \multicolumn{10}{|l|}{ Ocupación } \\
\hline No manual & 2 & $66,7(9,4-99,2)$ & 0,740 & 0 & 0,0 & 0,217 & 2 & $50,0(6,8-93,2)$ & 0,660 \\
\hline Manual & 30 & $63,8(49,0-78,6)$ & & 8 & $50,0(24,7-75,3)$ & & 38 & $60,3(47,7-73,2)$ & \\
\hline Labores del hogar & 6 & $75,0(34,9-96,8)$ & & 113 & $65,7(58,3-73,1)$ & & 119 & $66,1(58,9-73,3)$ & \\
\hline Otros & 49 & $57,6(46,6-68,7)$ & & 8 & $80,0(44,4-97,5)$ & & 57 & $60,0(49,6-70,4)$ & \\
\hline \multicolumn{10}{|l|}{ Estado civil } \\
\hline Soltero & 13 & $56,5(34,1-79,0)$ & 0,806 & 35 & $67,3(53,6-81,0)$ & 0,007 & 48 & $64,0(52,5-75,5)$ & 0,118 \\
\hline Casado-Unión libre & 53 & $63,1(52,2-74,0)$ & & 31 & $48,4(35,4-61,5)$ & & 84 & $56,8(48,4-65,1)$ & \\
\hline Viudo & 15 & $62,5(41,0-84,0)$ & & 50 & $75,6(64,7-86,9)$ & & 65 & $72,2(62,4-82,0)$ & \\
\hline Separado & 6 & $50,0(21,1-78,9)$ & & 13 & $76,5(50,1-93,2)$ & & 19 & $65,6(46,5-84,5)$ & \\
\hline \multicolumn{10}{|l|}{ Zona de residencia } \\
\hline Urbana & 76 & $58,9(50,0-67,8)$ & 0,152 & 119 & $67,2(60,0-74,4)$ & 0,044 & 195 & $63,7(58,2-69,3)$ & 0,526 \\
\hline Rural & 11 & $78,6(49,2-95,3)$ & & 10 & $45,5(22,4-68,5)$ & & 21 & $58,3(40,8-75,8)$ & \\
\hline \multicolumn{10}{|l|}{ Apoyo social } \\
\hline Normal & 60 & $55,0(45,2-64,8)$ & 0,011 & 91 & $59,9(51,7-68,0)$ & 0,008 & 151 & $57,9(51,7-64,0)$ & $<0,001$ \\
\hline Bajo & 27 & $79,4(64,4-94,5)$ & & 38 & $80,9(68,5-93,2)$ & & 65 & $80,2(71,0-89,5)$ & \\
\hline Total & 87 & $60,8(52,5-69,2)$ & ----- & 129 & $64,8(57,9-71,7)$ & ----- & 216 & $63,2(57,9-68,4)$ & ----- \\
\hline
\end{tabular}

*Pruebas Chi cuadrado de distribución de frecuencia y de diferencia de proporciones.

más del $85 \%$ pertenecen a estratos socioeconómicos bajos, en mayor proporción dedicados a las labores del hogar, especialmente las mujeres ( $86 \%$ ), aunque se destaca casi una tercera parte de los hombres dedicados a ocupaciones de menor cualificación (manuales). Más de la mitad de los hombres estaban casados o en unión libre, y una tercera parte de las mujeres eran viudas. Un $11 \%$ de la población adulta encuestada vive en zonas rurales de la ciudad y un $24 \%$ percibieron bajo apoyo social.

Teniendo en cuenta la prevalencia autopercibida de mala salud mental, según en GHQ12 (Tabla 2), los datos dan cuenta que esta fue mayor en población $\geq$ 75 años, tanto en hombres como en mujeres $(68,2 \%$; IC 95\%: 58,9-77,5). Se encontró un gradiente social importante, en cuanto al nivel educativo, puesto que a mejor nivel educativo la prevalencia de mala salud mental fue menor en ambos sexos y se encontraron diferencias significativas $(p<0,001)$. La prevalencia fue mayor en población de estratos bajos $(64,2 \%$; IC 95\%: 58,5-69,8), aunque no se encontraron diferencias significativas $(p=0,346)$. En el caso de los hombres la prevalencia de mala salud mental fue mayor en la zona rural (78,6\%; IC 95\%: 49,2-95,3) y para las mujeres en la zona urbana y se encontraron diferencias significativas $(67,2 \%$; IC 95\%: 60,0-74,4; $p=0,044)$. Las personas con bajo apoyo social presentaron mayor prevalencia de mala salud mental (80,2\%; IC 95\%: $71,0-89,5)$ y se encontraron diferencias significativas en ambos sexos $(p<0,001)$.

Por último, el análisis multivariado por regresión logística mostró que luego de ajustar por variables de confusión, la población adulta sin estudios de ambos sexos (ORa 7,61; IC 95\%: 2,49- 23,34), las mujeres viudas (ORa 2,78; IC 95\% 1,21-6,40) y los hombres y mujeres con bajo apoyo social (ORa 3,05; IC 95\%: 1,39$7,50)$, presentaron mayor riesgo de presentar mala salud mental y con asociación significativa respecto a sus contrapartes de referencia. En el modelo final, las mujeres tuvieron mayor riesgo de presentar mala salud mental, pero dicha asociación no fue significativa (ORa 1,23; IC 95\%: 0,76-1,97) Tabla 3. 
Tabla 3. Asociación de la mala salud mental con las características sociodemográficas en la población adulta mayor atendida en la E.S.E METROSALUD. Medellín, Colombia. $2013(n=342)$

\begin{tabular}{|c|c|c|c|c|c|c|}
\hline \multirow{2}{*}{ Características } & \multicolumn{2}{|c|}{ Hombres } & \multicolumn{2}{|c|}{ Mujeres } & \multicolumn{2}{|r|}{ Total $\pi$} \\
\hline & ORa & (IC 95\%) & ORa & (IC 95\%) & ORa & (IC 95\%) \\
\hline \multicolumn{7}{|l|}{ Nivel educativo* } \\
\hline Secundaria-Universitaria & 1,00 & ----- & 1,00 & ----- & 1,00 & ----- \\
\hline Primaria & 8,58 & $(1,77-41,65)$ & 3,64 & $(1,02-12,97)$ & 5,27 & $(2,04-13,62)$ \\
\hline Sin estudios & 9,91 & $(1,48-66,49)$ & 8,44 & $(1,71-41,61)$ & 7,61 & $(2,49-23,34)$ \\
\hline \multicolumn{7}{|l|}{ Estado civil ${ }^{\dagger}$} \\
\hline Casado-Unión libre & 1,00 & ----- & 1,00 & ----- & 1,00 & ----- \\
\hline Soltero & 0,84 & $(0,30-2,34)$ & 2,17 & $(0,96-4,93)$ & 1,39 & $(0,75-2,60)$ \\
\hline Viudo & 1,10 & $(0,38-3,15)$ & 2,78 & $(1,21-6,40)$ & 1,79 & $(0,94-3,40)$ \\
\hline Separado & 0,58 & $(0,14-2,45)$ & 3,63 & $(0,98-13,47)$ & 1,47 & $(0,60-3,64)$ \\
\hline \multicolumn{7}{|l|}{ Zona de residencia ${ }^{\ddagger}$} \\
\hline Urbana & 1,00 & ----- & 1,00 & ---- & 1,00 & ----- \\
\hline Rural & 2,02 & $(0,51-8,05)$ & 0,55 & $(0,21-1,47)$ & 0,87 & $(0,39-1,77)$ \\
\hline \multicolumn{7}{|l|}{ Apoyo social§ } \\
\hline Normal & 1,00 & ----- & 1,00 & ---- & 1,00 & ----- \\
\hline Bajo & 2,86 & $(1,12-7,34)$ & 3,23 & $(1,39-7,50)$ & 3,05 & $(1,65-5,66)$ \\
\hline Total" & 1,00 & ---- & 1,23 & $(0,76-1,97)$ & ----- & ----- \\
\hline
\end{tabular}

"Ajustada por edad, estrato socioeconómico, ocupación, estado civil, zona de residencia y apoyo social

† Ajustada por edad, nivel educativo, estrato socioeconómico, ocupación, zona de residencia y apoyo social

$\ddagger$ Ajustada por edad, nivel educativo, estrato socioeconómico, ocupación, estado civil y apoyo social

$\S$ Ajustada por edad, nivel educativo, estrato socioeconómico, ocupación, estado civil y zona de residencia

II Ajustada por edad, nivel educativo, estrato socioeconómico, ocupación, estado civil, zona de residencia y apoyo social

II Se incluye la variable sexo en el modelo total

\section{DISCUSIÓN}

En este estudio se identificaron, según los hallazgos, algunos factores sociodemográficos relacionados con la prevalencia de mala salud mental en adultos mayores atendidos en la red de prestación de servicios de salud pública de Medellín. Se observaron gradientes sociales en esta prevalencia según nivel educativo y un mayor riesgo de mala salud mental en las mujeres viudas y en las personas con bajo apoyo social. Aunque sin diferencias notables estadísticamente, el estrato socioeconómico, la edad y la zona de residencia fueron factores que influyeron en la prevalencia de mala salud mental en la población estudiada. Hasta donde alcanza nuestro conocimiento, este es uno de los primeros estudios que evalúa salud mental a nivel institucional y poblacional en un colectivo de especial vulnerabilidad como son los adultos mayores atendidos en esta red pública.

Los resultados no son fácilmente comparables con otros estudios debido a los instrumentos y escalas de medición utilizadas. Por ejemplo, la prevalencia de mala salud mental en los adultos mayores que acuden a la red sanitaria pública de la ciudad es mayor a la depresión reportada en un estudio realizado en 103 centros de bienestar del anciano ${ }^{(18)}$ (45,7\% teniendo en cuenta ambos sexos). Al comparar los resultados con la Encuesta Nacional de Demografía y Salud -ENDS
$2010^{(16)}$, la cual evalúa cada una de las dimensiones del test de Zung se encuentran síntomas relacionados con depresión y ansiedad en proporciones entre el 40 y $60 \%$ (más o menos concordantes con el estudio en Medellín). A nivel internacional, en un estudio en adultos mayores de 64 años en Guadalajara, México (26) (con la salvedad de que utilizan otras escalas para medir depresión), la prevalencia de síntomas depresivos fue del 19,7\% (menor a lo reportado en Medellín, aunque son poblaciones similares en el contexto social). Un estudio español que utilizó el GHQ-12 ${ }^{(13)}$, encontró una prevalencia de $9,4 \%$ en hombres y $21,8 \%$ en mujeres (la prevalencia en la población adulta mayor que acude a la red pública de prestación de servicios de salud fue del $61 \%$ en hombres y $65 \%$ en mujeres).

Los resultados encontrados ponen de manifiesto la situación de vulnerabilidad a la cual está expuesta la población adulta mayor de la ciudad de Medellín. Condición que se ve reflejada por la situación social en los barrios y comunas de la ciudad, el contexto sociopolítico y por la dificultad de implementación de políticas sociales y de salud para esta población ${ }^{(27)}$. A esta situación se suma el hecho de encontrar subgrupos que tienen una mayor vulnerabilidad, como por ejemplo las personas de menor nivel educativo y con bajo apoyo social. Las bajas oportunidades de desarrollo en un contexto social con profundas desigualdades sociales, se ven reflejadas en la situación de salud ${ }^{(28)}$, lo cual 
ha sido descrita en la literatura como la "paradoja de la desigualdad", donde existen grupos que están en mayor riesgo por las características sociales que comparten ${ }^{(29)}$. En cuanto al apoyo social, la literatura comenta cómo las personas que no cuentan con una red social de apoyo, dada por amigos, familiares o en algunos casos, por un apoyo institucional, tienen peor salud mental y mayor depresión ${ }^{(13,18)}$.

Conviene analizar la relación entre sexo y salud. Coindicen los hallazgos con otro estudio en la ciudad al encontrar una mayor sintomatología reportada en mujeres, aunque en ambas investigaciones no encontraron diferencias significativas con respecto a los hombres ${ }^{(18)}$. Estudios previos llaman la atención sobre la relación que existe entre la posición socioeconómica y el sexo, que coloca a las mujeres en una situación de especial vulnerabilidad. ${ }^{(30,31)}$. Los análisis multivariados encontraron una probabilidad aumentada de mala salud mental y estadísticamente significativa en las mujeres viudas. Un estudio realizado en Medellín sobre el sentimiento de soledad en población adulta mayor, muestra cómo se halló una mayor soledad conyugal en la mujer ${ }^{(32)}$. Este sentimiento de soledad, sumado a otras circunstancias relacionadas con el apoyo social, incrementa el riesgo de depresión y mala salud mental.

Aunque no se encontraron diferencias significativas en los análisis ajustados, la prevalencia de mala salud mental fue mayor en los hombres que viven en áreas rurales. Al respecto, es importante comentar los roles que cumplen ambos sexos y sus representaciones sociales ${ }^{\left({ }^{3}\right)}$. Muchos de los hombres todavía laboran en ocupaciones de tipo manual, y no cuentan con beneficios sociales, como el derecho a la jubilación. Esto se constituye en un importante determinante de acceso a los servicios de salud. Estudios llevados en áreas rurales, que incluyen adultos mayores, muestran como esta población experimenta depresión y barreras de acceso a servicios de salud mental (diagnóstico y tratamiento) ${ }^{(34)}$.

La prevalencia de mala salud mental en la población adulta mayor de Medellín fue mucho mayor a lo encontrado en la población general de Colombia. Según los últimos datos disponibles a nivel poblacional (2003) ${ }^{(35)}$, la frecuencia de trastornos en el curso de vida según DSM-IV Colombia es $40,1 \%$, esto para el grupo poblacional de 18 a 65 años. Es importante mencionar que los problemas de salud mental inician entre los 9 y 23 años. Existen importantes factores culturales, sociales y económicos asociados a la prevalencia de estos problemas que han tenido repercusión en fenómenos epidemiológicos de cohorte, evidenciando en la población adulta mayor problemas que pueden haberse originado años atrás. Esto puede explicarse desde la epidemiología social; las desigualdades sociales que impactan en la situación de salud tienen un componente histórico importante y se han incorporado en la población de forma acumulativa (36).

Es importante comentar las fortalezas y limitaciones de este estudio. Se contó con una muestra suficiente de adultos mayores representativa de las unidades hospitalarias y centros de salud de la red prestadora de servicios de salud pública de la ciudad de Medellín. De igual forma, la utilización de instrumentos ya validados permitió hacer comparaciones con algunos estudios a nivel internacional. Se realizó control de calidad en los cuestionarios, lo cual favorece su confiabilidad. Como limitaciones conviene mencionar que este estudio se basó en datos de un proyecto macro que pretendió identificar los factores determinantes de la calidad de vida y su impacto en la salud bucal en la población adulta mayor que acude a los servicios de consulta general y especializada en la E.S.E Metrosalud de Medellín, desde una perspectiva de género. Esta investigación incluyó variables de salud general y mental. Para la determinación del tamaño muestral, la variable salud mental fue secundaria a la variable calidad de vida y esto es importante a la hora de extrapolar los resultados. No obstante, haciendo cálculos muestrales adicionales en el programa EPIINFO, tomando datos de prevalencia de algunos problemas de salud mental para Colombia ${ }^{(16)}$ y para Medellín (18) (teniendo en cuenta que utilizan otros indicadores para tamizaje), el tamaño oscila entre 240 y 366 adultos mayores, los cuales se ajustan al muestreo utilizado en el estudio de Metrosalud. Es Importante destacar que la naturaleza transversal del estudio no permite establecer causalidad en las relaciones y asociaciones encontradas.

El cuestionario GHQ-12, al ser un instrumento de percepción, debe interpretarse con cautela, por su naturaleza de prueba tamiz y no como instrumento diagnóstico. En cuanto al punto de corte utilizado, se optó por el usado por Lahuerta et al (13), ya que era una población no institucionalizada que compartía características similares al estudio de Medellín y permitía comparabilidad internacional. No obstante, la investigación debe ser complementada con estudios específicos en salud mental que permitan un abordaje poblacional, la validación del instrumento interna y externa con entrevista psicológica o psiquiátrica y profundizar en trastornos específicos (emocionales o cognitivos), esto con el fin de tener mayor fiabilidad en los resultados. Sin embargo, teniendo en cuenta la naturaleza epidemiológica del estudio, la capacidad del instrumento para detectar población en riesgo ya ha sido validada en estudios previos ${ }^{(25)}$, y estos indicadores de salud autopercibida son usados ampliamente, trascendiendo el paradigma biomédico tradicional de ver el proceso salud-enfermedad (37). 
Conviene complementar los resultados de este estudio, con la población adulta mayor que consulta al régimen contributivo en el Sistema General de Seguridad Social en Salud (capacidad de pago), con el fin de ofrecer un panorama más amplio de la situación de salud de este grupo poblacional. Por otro lado, se requiere profundizar sobre factores contextuales, económicos, políticos y sociales que inciden en la situación de salud. Nuevas investigaciones a través de metodologías cualitativas podrían ser útiles en la identificación de situaciones de desigualdad a través de la percepción de diversos actores que prestan servicios de salud a la población adulta mayor, y a través del estudio de las percepciones de los adultos mayores sobre los determinantes que afectan su situación de salud mental.

En conclusión, los resultados de este estudio evidencian la necesidad de establecer sistemas de seguimiento y evaluación epidemiológica de diversos indicadores de salud mental específicos para la población adulta mayor. Si bien existe la Ley de Protección Social Para la Población Adulta Mayor en Colombia (251/2008) (38), se debe continuar reforzando las políticas en promoción y prevención y estrategias de intervención en salud desde el enfoque de los determinantes sociales ${ }^{\left({ }^{39)}\right.}$ que contribuyan a ofrecer un ambiente favorable para la salud mental y las políticas sociales que son necesarias para fortalecer las redes sociales de apoyo, y permitir la disminución de las desigualdades sociales en salud y, por ende, la vulnerabilidad para este grupo poblacional.

Agradecimientos: el grupo de investigación agradece al grupo de adultos mayores que participaron en el estudio y al grupo de profesionales y estudiantes que participaron en el trabajo de campo en la realización de encuestas y exámenes clínicos.

Contribuciones de autoría: AAAS participó en la concepción y diseño del artículo, y realizó los análisis e interpretación de los datos con la participación de APL y EJMG. AAAS realizó la primera versión y redacción del manuscrito. Todos los autores participaron en la redacción crítica del artículo y aprobación de la versión final.

Fuentes de financiamiento: Empresa Social del Estado Metrosalud. Medellín (Código: C02-E11-L3-01)

Conflictos de interés: los autores declaran no tener conflitos de interés.

\section{REFERENCIAS BIBLIOGRÁFICAS}

1. Peláez M. La construcción de las bases de la buena salud en la vejez: situación en las Américas. Rev Panam Salud Public. 2005;17(5/6):299-302.

2. Cardona Arango D. Envejecimiento poblacional: reto a la salud pública. CES Salud Pública. 2013;4(2):82-3.

3. Comisión Económica para América Latina (CEPAL). Población, envejecimiento y desarrollo. Documento LC/G.2235(SES.30/16). San Juan: CEPAL; 2004.

4. Departamento Administrativo Nacional de Estadística (DANE). Estudios postcensales 7: Proyecciones nacionales y departamentales de población 2005- 2020. Bogotá: DANE; 2010

5. Alcaldía de Medellín. Plan de Desarrollo "Medellín, un hogar para la vida" 2012 - 2015. Medellín: Alcaldía; 2012.

6. Cardona D, Estrada A, Agudelo H. Medellín envejece a pasos agigantados. Rev Fac Nac Salud Pública. 2004; 22 (4): 7-19

7. Cardona D, Estrada A, Agudelo HB. Calidad de vida y condiciones de salud de la población adulta mayor de
Medellín. Biomédica. 2006;26(2):20615.

8. Cardona D. Comparativo de la calidad de vida del adulto mayor. Medellín, 2008. Rev Fac Nac Salud Publica. 2010;28(2):149-60.

9. Cardona Jiménez JL, Villamil Gallego MM, Henao Villa E, Quintero Echeverri Á. El sentimiento de soledad en adultos. Medicina UPB. 2013;32(1):9-19.

10. Alonso Galbán P, Sansó Soberats FJ, Díaz-Canel Navarro AM, Carrasco García M, Oliva T. Envejecimiento poblacional y fragilidad en el adulto mayor. Rev Cubana Salud Publica. 2007;33(1):1-17. http://scielo.sld.cu/ pdf/rcsp/v33n1/spu10107.pdf

11. Sánchez Gil IY, Pérez Martínez VT. El funcionamiento cognitivo en la vejez: atención y percepción en el adulto mayor. Rev Cubana Med Gen Integr [Internet]. 2008 [citado el 5 de enero de 2015];24(2):[17 p.]. Disponible en: http://scielo.sld.cu/pdf/rcsp/v33n 1/ spu10107.pdf

12. Dorantes-Mendoza G, Ávila-Funes JA, Mejía-Arango S, GutiérrezRobledo LM. Factores asociados con la dependencia funcional en los adultos mayores: un análisis secundario del Estudio Nacional sobre Salud y Envejecimiento en México, 2001. Rev Panam Salud Publ. 2007;22(1):1-11.

13. Lahuerta C, Borrell C, Rodríguez-Sanz M, Pérez K, Nebot M. La influencia de la red social en la salud mental de la población anciana. Gac Sanit. 2004;18(2):83-91

14. Organización Mundial de la Salud. Centro de Prensa. La salud mental y los adultos mayores. Nota descriptiva 381, Septiembre de 2013. Ginebra: OMS; 2013.

15. Riedel-Heller SG, Busse A, Angermeyer MC. The state of mental health in oldage across the 'old' European Union-a systematic review. Acta Psychiatr Scand. 2006 May;113(5):388-401.

16. Profamilia. Encuesta Nacional de Demografía y Salud ENDS Colombia 2010. Bogotá: Profamilia- Ministerio de Protección Social; 2010.

17. Cardona-Arango D, EstradaRestrepo A, Chavarriaga-Maya LM, Segura-Cardona AM, OrdoñezMolina J, Osorio-Gómez JJ. Apoyo social dignificante del adulto mayor 
institucionalizado. Medellín, 2008. Rev Salud Publica (Bogota). 2010;12(3):414-24.

18. Estrada A, Cardona D, Segura AM, Ordóñez J, Osorio JJ, Chavarriaga LM. Síntomas depresivos en adultos mayores institucionalizados y factores asociados. Univ Psychol. 2013;12(1):81-94.

19. Metrosalud.gob.co [Internet]. Medellín: Empresa Social del Estado (ESE) Metrosalud [actualizado el 12 de noviembre de 2015; citado el 02 de setiembre de 2014]. Disponible en: http://www.metrosalud.gov.co/inter/ joomla/

20. Montero J, Yarte JM, Bravo M, LópezValverde A. Oral health-related quality of life of a consecutive sample of Spanish dental patients. Med Oral Patol Oral Cir Bucal. 2011;16(6):e810-5.

21. Meneses-Gómez EJ. Salud bucodental y calidad de vida oral en los mayores. Tesis para obtener el grado de Doctor. Facultad de Odontología, Universidad Complutense de Madrid. Madrid, España. 2010.

22. República de Colombia. Departamento Administrativo Nacional de Estadística (DANE). Clasificación internacional uniforme de ocupaciones adaptada para Colombia. Bogotá: DANE; 2005.

23. Broadhead WE, Gehlbach SH, de Gruy FV, Kaplan BH. The DukeUNC functional social support questionnaire. Measurement of social support in family medicine patients. Med Care. 1988 Jul;26(7):709-23.

24. Bellón JA, Delgado A, Luna JD, Lardelli P. Validez y fiabilidad del cuestionario de apoyo social funcional
Duke-UNC-11. Aten Prim. 1996 Sep;18(4):153-62.

25. Goldberg D, Williams P. Cuestionario de salud general GHQ: guía para el usuario de las distintas versiones. Barcelona: Masson; 1996.

26. Urbina Torija JR, Flores Mayor JM, García Salazar MP, Torres Buisán L, Torrubias Fernández RM. Síntomas depresivos en personas mayores: Prevalencia y factores asociados. Gac Sanit. 2007;21(1):37-42.

27. Cardona Arango D, Segura Cardona AM. Políticas de salud pública aplicadas al adulto mayor en Colombia. Rev Esp Geriatr Gerontol. 2011;46(2):96-9.

28. Ministerio de la Protección Social. Análisis de la Situación de Salud en Colombia, 2002-2007. Tomo IV: Análisis de desigualdades e inequidades en salud. Bogotá: Imprenta Nacional de Colombia; 2011.

29. Frohlich KL, Potvin L. Transcending the known in public health practice: the inequality paradox: the population approach and vulnerable populations. Am J Public Health. 2008 Feb;98(2):216-21. doi: 10.2105/ AJPH.2007.114777.

30. Kunkel SR, Atchley RC. Why gender matters: being female is not the same as not being male. Am J Prev Med. 1996 Sep-Oct;12(5):294-6.

31. Macintyre S, Hunt K. Socio-economic Position, Gender and Health: How Do They Interact?. J Health Psychol. 1997 Jul;2(3):315-34. doi: $10.1177 / 135910539700200304$.

32. Cardona Jiménez JL, Villamil Gallego MM, Henao Villa E, Quintero
Echeverri Á. El sentimiento de soledad en adultos. Medicina UPB. 2013;32(1):9-19.

33. Colom Bauzá J. Vejez, representación social y roles de género. Educació i Cultura. 1999; 12:47-56.

34. Bocker E, Glasser M, Nielsen K, Weidenbacher-Hoper V. Rural older adults' mental health: status and challenges in care delivery. Rural Remote Health. 2012;12:2199.

35. Posada JA. La salud mental en Colombia. Biomédica. 2013;33(4):497-8.

36. Krieger N. Embodiment: a conceptual glossary for epidemiology. J Epidemiol Community Health. 2005 May;59(5):350-5.

37. Segovia J, Bartlett RF, Edwards AC. An empirical analysis of the dimensions of health status measures. Soc Sci Med. 1989;29(6):761-8.

38. Colombia, Congreso de la República. Ley 1251 (2008) "por la cual se dictan normas tendientes a procurar la protección, promoción y defensa de los derechos de los adultos mayores". Bogotá: Diario Oficial No. 47.186; 2008.

39. Colombia, Ministerio de Salud y Protección Social. Plan Decenal de Salud Pública, PDSP, 2012- 2021. Bogotá D.C.: MinSalud; 2013.

Correspondencia: Andrés A. Agudelo Suárez Dirección: Calle 70 No. 52-21. Medellín, Colombia.

Teléfono: +5742196740, +5742196741, $+573006812530$

Correoelectrónico:oleduga@gmail.com 